Check for updates

Cite this: Chem. Sci., 2018, 9, 8375

๑ All publication charges for this article have been paid for by the Royal Society of Chemistry

\title{
Selective parallel G-quadruplex recognition by a NIR-to-NIR two-photon squaraine $\uparrow$
}

\author{
Vincenzo Grande, $\neq^{\mathrm{ab}}$ Chia-An Shen, $\neq^{\mathrm{a}}$ Marco Deiana, ${ }^{\mathrm{c}}$ Marta Dudek, ${ }^{\mathrm{c}}$ \\ Joanna Olesiak-Banska, (D) Katarzyna Matczyszyn (D) *c and Frank Würthner (D) *ab
}

Fluorescence imaging probes for specific G-quadruplex (G4) conformations are of considerable interest in biomedical research. Herein, we present the synthesis and the binding properties of a new water-soluble near-infrared (NIR) amphiphilic squaraine dye (CAS-C1) which is capable of selective detection of parallel over non-parallel and non G4 topologies. The striking changes in its linear optical response upon binding to parallel G4s give rise to high fluorescence quantum yields $\left(\Phi_{f} \approx 0.7\right)$ and one-photon molecular brightness in the far-red-NIR region. The outstanding recognition process of CAS-C1 for parallel G4s via end-stacking provides binding constants in the nanomolar regime $\left(K_{\mathrm{b}}=10^{7}\right.$ to $\left.10^{8} \mathrm{M}^{-1}\right)$ awarding it as one of the most potent parallel G4 binders currently available. Moreover, the CAS-C1-parallel G4 system exhibits large two-photon absorption (TPA) cross-sections and molecular brightness in the second NIR biological transparency window $(\lambda \approx 1275 \mathrm{~nm})$, making it an ideal candidate for NIR-to-NIR ultrasensitive two-photon procedures.

Received 29th June 2018

Accepted 26th August 2018

DOI: $10.1039 / \mathrm{c} 8 \mathrm{sc} 02882 \mathrm{f}$

rsc.li/chemical-science

majority of the small optical imaging probes for G4 detection are commonly photoexcited by relatively high-to-moderate energy light (330-600 nm $)^{\mathbf{1 4 , 1 5}}$ in order to generate the emission between the visible and far-red region of the electromagnetic spectrum. Such energies, in particular UV or blue, are in themselves deleterious to cells as they can irreversibly damage DNA and/or induce formation of cytotoxic reactive oxygen species. ${ }^{16}$ Furthermore, their excitation and emission wavelengths are confined outside the first and second near-infrared (NIR) biological transparency windows (BTWs, NIR-I: 700-950 nm and NIR-II: 1000-1350 $\mathrm{nm})^{17}$ where absorption and scattering from tissues and oxy/deoxygenated blood is significant, limiting their relevance to $2-\mathrm{D}$ cell cultures and precluding their use in more realistic diagnosis environments, such as in thick tissues or in vivo models. The lack of biocompatible fluorescent organic probes in the BTWs has prevented the use of these highly sensitive spectral ranges for in-depth imaging. ${ }^{17}$ In addition, the dynamic nature of the G4 structure enable a range of different topologies (e.g. parallel, hybrid and antiparallel) to potentially form under different physiological conditions, making molecular recognition particularly arduous. In our research on G4-probes, we have recently shown that a dicyanovinyl squaraine dye (SQgI, Fig. 1a) ${ }^{\mathbf{1 8}}$ could behave as G4-specific NIR light-up probe. In the complexed state, SQgl is emissive at $700 \mathrm{~nm}$ upon excitation at $661 \mathrm{~nm}$ through one-photon absorption (OPA) with an impressive quantum yield of $\Phi_{\mathrm{F}} \approx 0.6$. The probe exhibited good selectivity of G4 over duplex, with the dicyanovinyl squaraine dye capable to impart outstanding selectivity over non-G4 structures and remarkable specificity toward parallel G4 topologies. However, this probe presented inherent optical, practical and affinity

\footnotetext{
aniversität Würzburg, Institut für Organische Chemie, Am Hubland, 97074 Würzburg, Germany. E-mail: wuerthner@uni-wuerzburg.de

Würzburg, Theodor-Boveri-Weg, 97074 Würzburg, Germany

${ }^{c}$ Advanced Materials Engineering and Modelling Group, Faculty of Chemistry, Wroclaw University of Science and Technology, Wybrzeze Wyspianskiego 27, 50-370 Wroclaw, Poland.E-mail: katarzyna.matczyszyn@pwr.edu.pl

$\dagger$ Electronic supplementary information (ESI) available: Synthesis, titration experiments, optical spectra, NMR and HRMS spectra. See DOI $10.1039 / \mathrm{c} 8 \mathrm{sc} 02882 \mathrm{f}$

\$ These authors contributed equally.
} 
a)

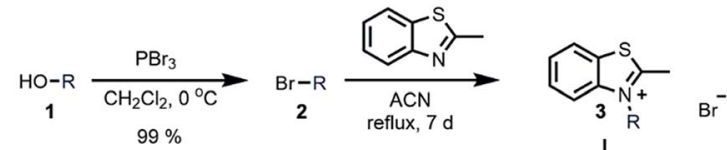

$99 \%$ non-parallel G4 structures (right).

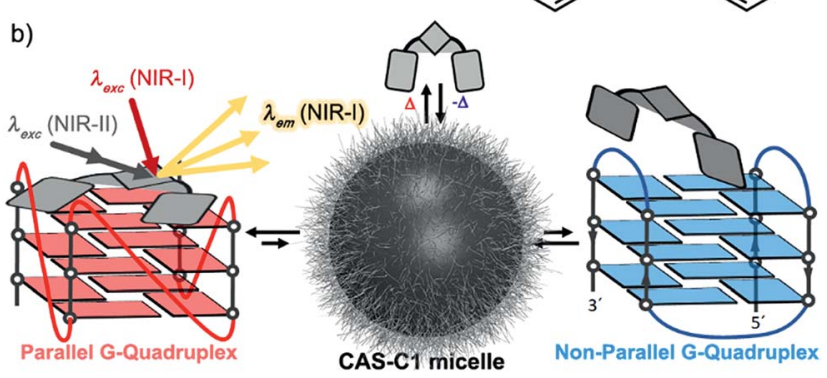

Fig. 1 (a) Synthetic route to CAS-C1. (b) Schematic illustration of the multiphotonic selective detection mechanism: the squaraine shows amphiphilic assembly in water into micelles. The formation of a stable complex with parallel G-quadruplex (left) is able to displace the squaraine from the micellar aggregate and trigger the fluorescent emission in the NIR-I biological window upon both one-photon excitation in the NIR-I and two-photon excitation in the NIR-II. CASC1 bestows high selectivity of interactions over non-quadruplex and

limitations including: (i) moderate solubility, (ii) moderate binding constants $\left(K_{\mathrm{b}}\right.$ values comprised between $10^{5}$ to $10^{6}$ $\left.\mathrm{M}^{-1}\right)^{18-20}$ and (iii) excitation wavelengths outside the BTWs. Thus, we speculated that exploring the chemical space around the squaraine core should enable optimization of both optical and DNA binding performances and we decided to decorate the dicyanovinylene squaraine moiety with bulkier water-soluble brush-type groups.

Herein, we present the first example of a water-soluble amphiphilic squaraine dye (hereafter CAS-C1, Fig. 1a) exhibiting G4-selective fluorescence light-up upon photoexcitation both within the NIR-I $\left(\lambda_{\max } \approx 720 \mathrm{~nm}\right.$ and $\left.\Phi_{\mathrm{F}} \approx 0.7\right)$ through OPA, and within the NIR-II (two-photon absorption crosssection, $\sigma_{2}$, of $\approx 300 \mathrm{GM}$ and two-photon molecular brightness, $\sigma_{2} \times \Phi_{\mathrm{F}}$, of $\approx 200 \mathrm{GM}$ at $1275 \mathrm{~nm}$ ) through TPA. Moreover, the unprecedented nonlinear optical properties of CAS-C1 are combined with the outstanding selectively for parallel over non-parallel G4 architectures and duplex morphologies of any length and composition awarding it as the best two-photon parallel G4-specific probe currently available.

\section{Results and discussion}

\section{Design and synthesis}

With the aim to increase the binding selectivity and the solubility of ligand SQgl, we decorated the dicyanovinylene squaraine core scaffold with brush substituents carrying six triethylene glycol chains (synthesis and characterization in ESI). The massive presence of oligoethylene glycol chains is not only beneficial for the solubility of the ligand, but it is also known to strengthen the affinity of the ligand toward the parallel G4s upon noncovalent interactions within the grooves. This approach is reported in the literature and has been exploited in the design of selective G4-ligands. ${ }^{21-23}$ The molecular design of dicyanovinyl squaraine revealed a suitable choice in terms of affinity and selectivity with G4s. ${ }^{18}$ Additionally, the donoracceptor-donor (D-A-D) nature of the chromophores ${ }^{24}$ appeared very promising for multi-photon absorption processes at long-infrared wavelengths. The synthesis of the newly designed squaraine according to the route shown in Fig. 1a starts from the reported brush precursor 1 that was brominated to give the corresponding bromide 2 , which underwent a nucleophilic substitution with methyl-benzothiazole to give 3 . Following the reported synthetic procedure for dicyanovinyl squaraines, ${ }^{24}$ CAS-C1 was obtained after condensation of the squaric ester 4 with two equivalents of methyl-benzothiazolium 3. Squaraine CAS-C1 revealed fairly good solubility in various organic solvents. The absorption and emission spectra in these solvents revealed a moderate negative solvatochromism (Fig. S2 $\dagger$ ) and can be assigned to monomeric species (Fig. S1†).

\section{Aggregation in water}

In aqueous solution, CAS-C1 absorption spectra reveal a different band shape and a strongly blue-shifted absorption maximum at $632 \mathrm{~nm}$ with an apparent extinction coefficient $\bar{\varepsilon}_{\max }=6.2 \times 10^{4} \mathrm{M}^{-1}$ (Fig. 2a, red line) which are first evidences for an $\mathrm{H}$-type exciton coupling between aggregated dyes. ${ }^{25}$

Atomic force microscopy (AFM) revealed the presence of small particles with an irregular diameter of $10-30 \mathrm{~nm}$ and a height of $0.6-3.0 \mathrm{~nm}$ (Fig. 2b) which suggests the formation of
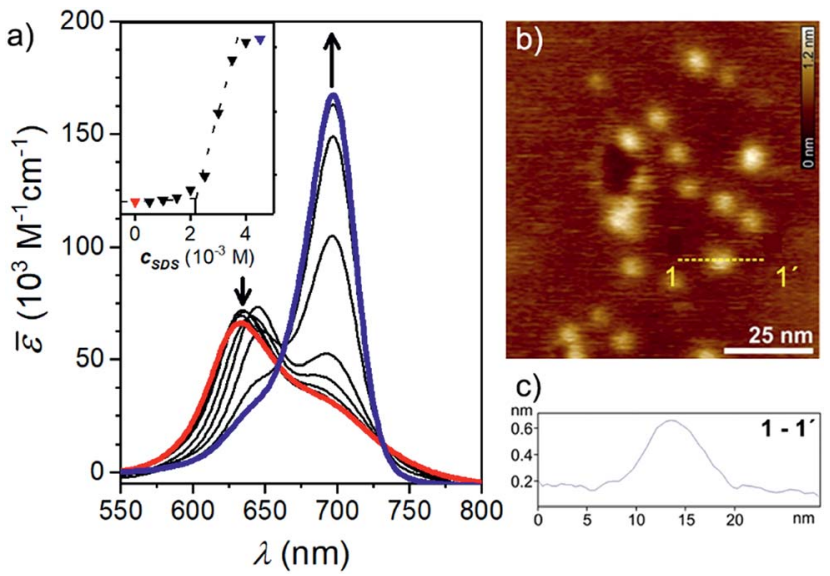

Fig. 2 (a) UV/Vis absorption spectra of an aqueous CAS-C1 solution (c $=3.0 \times 10^{-6} \mathrm{M}, 25^{\circ} \mathrm{C}$, red line) and upon addition of increasing amounts of SDS. The inset shows the incorporation of the squaraine dye in the SDS micelle upon addition of the surfactant with a critical concentration of $2.1 \times 10^{-3} \mathrm{M}$. (b) AFM height images on mica after spin coating the CAS-C1 aqueous solution $\left(3.0 \times 10^{-6} \mathrm{M}\right)$. $Z$ scale is $1.2 \mathrm{~nm}$. (c) Cross-section from yellow dashed lines $1-1^{\prime}$. 
micellar structures. The blue-shifted absorption band indicates a co-facial stacking of CAS-C1 molecules, most likely in an antiparallel fashion in order to cancel the dyes' large dipole moment. ${ }^{26}$ Such arrangement explains well the strong H-type excitonic coupling and the quenched fluorescence emission. Due to the massive presence of ethylene glycol chains, CAS-C1 is particularly suited to form such micellar structure where the hydrophilic parts are predominantly directed to the outside (Fig. 1b).

Upon heating to $95{ }^{\circ} \mathrm{C}$, CAS-C1 micellar aggregates become disfavored for entropic reasons leading to a new band at $676 \mathrm{~nm}$ with isosbestic point at $653 \mathrm{~nm}$. These changes are reversible and are ascribed to the formation of the monomeric species in water, with the typical fluorescence emission peaked at $699 \mathrm{~nm}$ (Fig. S3A $\dagger$ ). Global fitting of the temperature-dependent measurement in the range $550-750 \mathrm{~nm}$ is in good agreement with an isodesmic aggregation mechanism (Fig. S3B $\dagger$ ). Despite the fact that the pure monomeric state is not fully reached in water, the extrapolation of the fitting at $676 \mathrm{~nm}$ predicts a reasonable value for monomeric CAS-C1 of $\bar{\varepsilon}_{\max }=9.0 \times$ $10^{4} \mathrm{M}^{-1} \mathrm{~cm}^{-1}$. In accordance with the disaggregation model, a fluorescence emission in water lights-up at $699 \mathrm{~nm}$ (Fig. S3A $\dagger$, dotted line). In contrast, the emission profile of CAS-C1 in its aggregated form is fully quenched due to nonradiative relaxation pathways in its $\mathrm{H}$-aggregate state. In order to investigate the photophysical properties of the excited states, time-correlated single photon counting (TCSPC) measurements of CAS-C1 in water at $80{ }^{\circ} \mathrm{C}$ were performed. The time-dependent decay fitted better with the biexponential function (eqn S2, ESI $\dagger$ ) suggesting the coexistence of two different species: one with longer lifetime $\left(\tau_{1}=4.76 \mathrm{~ns}\right)$, consistently ascribed to the excited state of the free monomer in solution; the other one with shorter lifetime ( $\tau_{2}=1.35 \mathrm{~ns}$ ), ascribed to the species aggregated in the micellar structure (Fig. S4 $\dagger$ ). The dramatic decrease of the lifetime and emission intensity is, therefore, in accordance with the formation of additional radiationless pathways in the aggregated form.

A more efficient approach to disassemble CAS-C1 into monomeric dyes is provided by the addition of surfactant molecules. Thus, we performed titration with sodium dodecyl sulfate (SDS) (Fig. 2a). The surfactant affects moderately the formation of the micelles at lower SDS concentration. Above the critical concentration of $2.1 \times 10^{-3} \mathrm{M}$, SDS induces a marked breakup of CAS-C1 micelles by dissolving the CAS-C1 monomer within the SDS micelles (Fig. 2a).

\section{G4 binding}

In a similar fashion to the disaggregation induced by SDS, molecular recognition of CAS-C1 to suitable $\pi$-surfaces should also cause the disassembly of the micelle. This idea prompted us to study the recognition process of the squaraine toward a large panel of biologically relevant $\mathrm{G} 4$ topologies such as those found in the human telomeres (Tel-22) and minisatellite (Ceb25), in the proto-oncogene involved in the tumorigenesis of urinary bladder cancer (hRAS1), in the promoter region of myc, kit, KRAS, bcl2, and vav genes (c-myc, c-kit2, c-kit87up, KRAS 32,
KRAS 22, bcl2 VAV1), in the vascular endothelial growth factor (VEGF) gene, in the telomeric transcripts (TERRA) and the thrombin-binding DNA aptamer (TBA) (see ESI $\dagger$ for details concerning the G4 topologies and their folding process). Indeed, upon addition of parallel G4s (e.g. VAV-1, c-myc and VEGF) the disappearance of the absorption band of CAS-C1 aggregate along with the formation of a new bathochromically shifted band centered at $\approx 700 \mathrm{~nm}$ indicate the formation of G4 (parallel):CAS-C1 complexes (Fig. 3, S6 and S7†). Concomitant with absorption changes, the steady-state emission of the squaraine fluorophore upon excitation at $\lambda_{\text {exc }}=660 \mathrm{~nm}$, almost fully quenched in its aggregated unbound state $\left(\lambda_{\mathrm{em}}=698 \mathrm{~nm}\right)$, lights-up in the NIR-I at $\lambda_{\mathrm{em}} \approx 720 \mathrm{~nm}$ (Fig. 3a, S8 and S9 $\dagger$ ).

Notably, both the absorption and the emission bands are $\approx$ $20 \mathrm{~nm}$ redshifted compared to the spectrum of the monomeric squaraine in water (Fig. S3†). This is a clear indication of a direct interaction of the cyanine $\pi$-surface of CAS-C1 with the guanines of the parallel quadruplexes. The excitation spectra and the lifetime decays recorded for CAS-C1 in the presence of G4s indicate that the strong photoluminescence observed arises mainly from the CAS-C1 : G4 complex through geometrical and structural restrictions which inhibit the nonradiative relaxation pathways, hence the increased lifetime and $\Phi_{\mathrm{F}}$ (Fig. S10-S12 $\dagger$ ). TCSPC decay trace of the CAS-C1 : G4 excited states (Fig. S1 and $\mathrm{S} 12 \dagger)$ showed the presence of only one species, ascribed to the complexed squaraine, in accordance with the monoexponential function (eqn S1, ESI $\dagger$ ). The singlet state lifetimes obtained by fitting of time-resolved fluorescence decays of the CAS-C1 : G4 complexes are generally longer than the ones in organic solvents and comprised in the range of 5-6 ns (Fig. S2B $\dagger$ ). This relatively long lifetimes with outstanding $\Phi_{\mathrm{F}}$ in the $700-800 \mathrm{~nm}$ range makes CAS-C1 particularly intriguing candidate as a fluorescence lifetime-based probe for G4 live-cell visualization by fluorescence lifetime imaging microscopy (FLIM).

Comparison of absorption and fluorescence responses between parallel and antiparallel G4s as well as single- and double-stranded DNA of any length and composition indicates a clear-cut binding preference of CAS-C1 for G4 parallel topologies (Fig. 3b). This was further confirmed by duplex DNA competition titration assays (Fig. S19 and S20 †) which revealed very modest interference of interaction with c-myc and VAV-1, even in the presence of 50 eq. of the $d s$-DNA.

It turned out that probe accommodation within the hydrophobic G4 scaffolds exert changes in the polarity of the surrounding that well-match a $E_{\mathrm{T}}{ }^{N}$ value of 0.6 (Fig. S2 $\dagger$ ). The optical changes arising in the absorption and emission profiles of the CAS-C1-parallel G4 systems result in excellent one-photon brightness (defined as the product $\varepsilon \times \Phi_{\mathrm{F}}$ ) in the NIR region with values comprised between 56 and $84 \times 10^{3} \mathrm{M}^{-1} \mathrm{~cm}^{-1}$ (Table 1).

As suggested by recent publications, ${ }^{27-29}$ the issue of the binding stoichiometry of the complexes in solution was assessed by Job's plot titration assays (Fig. S13-S15†), the tangent method and a global fitting based on both the $2: 1,1: 1$ and $1: 2$ binding models (Fig. S16-S18 $\dagger$ ). If compared with the previously reported SQgl, CAS-C1 also showed multiple binding with the G4s, except for sG4, VAV-1 and c-myc. 


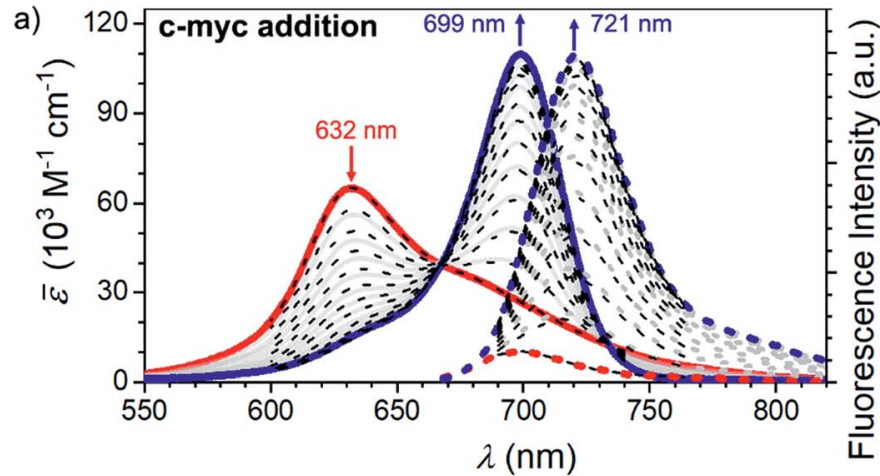

b)

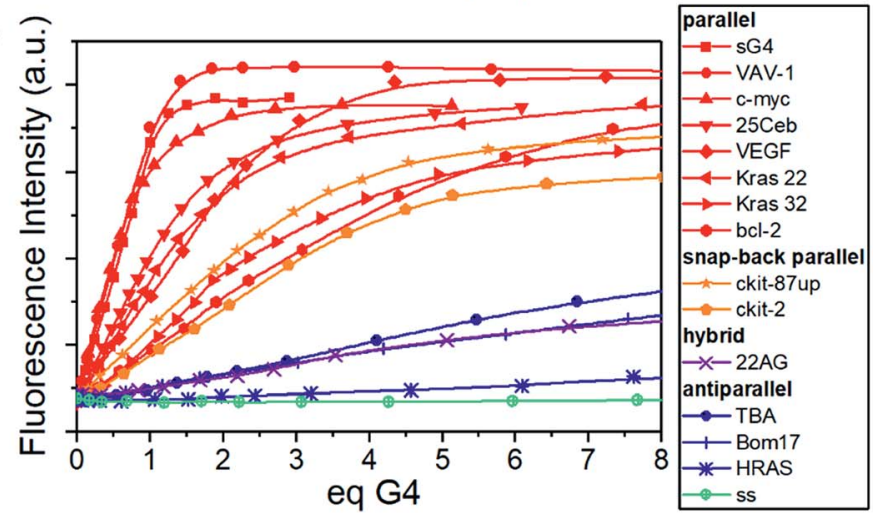

c)

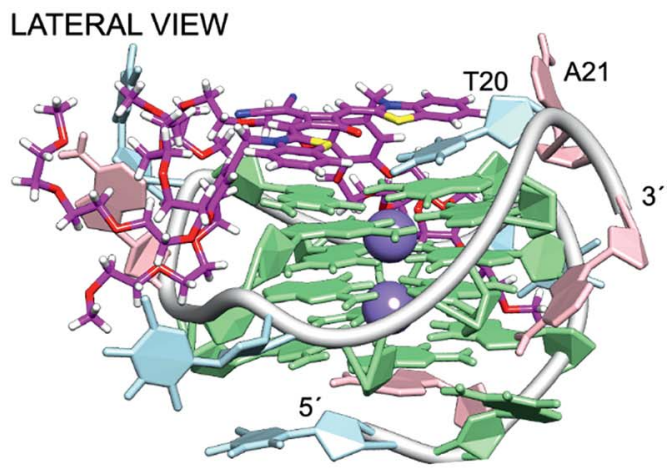

d)

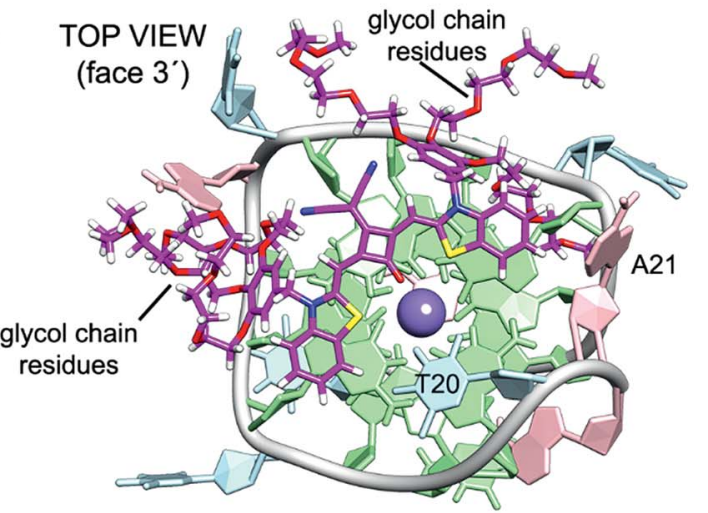

Fig. 3 (a) Visible/NIR absorption (solid lines) and fluorescence spectra (dotted lines, excitation on the isosbestic point at $660 \mathrm{~nm}$ ) of a CAS-C1 buffered solution in water $\left(1.66 \times 10^{-6} \mathrm{M}\right.$, TRIS buffer $\left.\mathrm{c}=10 \mathrm{mM}, \mathrm{pH}=7.2, \mathrm{KCl} \mathrm{c}=100 \mathrm{mM}\right)$ upon addition of $\mathrm{c}-\mathrm{myc} \mathrm{G} 4$ at $25^{\circ} \mathrm{C}$ (red and blue lines correspond to the spectra at 0 eq. and 3.4 eq. respectively. Superimposed black dotted line results from a global fitting with a $1: 1$ binding model $\left(K_{b}=9.7 \times 10^{6} \mathrm{M}^{-1}\right)$. (b) Fluorescence titrations of the CAS-C1 buffered solution in water upon addition of various $\mathrm{G} 4 \mathrm{~s}$ sequences at $25^{\circ} \mathrm{C}$ : the plots show a clear-cut preference of the squaraine for the parallel G4s (in red). (c and d) Docking lowest energy binding models for CAS-C1 with c-myc (score function $\Delta E=-5.9 \mathrm{kcal} \mathrm{mol}^{-1}$ ).

A detailed quantitative binding data analysis that takes into account $1: 1$ and $1: 2$ CAS-C1 : G4 stoichiometries with associated (anti-)cooperative effects indicates that CAS-C1 exhibits higher binding constants for parallel topologies such as c-myc (Fig. 3c and d) with respect to antiparallel and non-G4 topologies with values that exceed by two-orders of magnitude those for the previously reported squaraine SQgI (see ESI $\dagger$ for details concerning the quantitative binding data analysis). ${ }^{18}$ These results are ascribed to the highly accessible parallel G-quartets which, being devoid of adjacent lateral or diagonal loops, provide better $\pi$-stacking platforms for the accommodation of the squaraine core, thereby resulting in a better planarity and

Table 1 Summary of the spectroscopic properties and the binding constants of the complexes of CAS-C1 with various G4s ${ }^{a}$

\begin{tabular}{|c|c|c|c|c|c|c|c|c|}
\hline G-quadruplex & $\begin{array}{l}\lambda_{\max } \\
(\mathrm{nm})\end{array}$ & $\begin{array}{l}\varepsilon_{\max } \\
\left(10^{3} \mathrm{M}^{-1} \mathrm{~cm}^{-1}\right)\end{array}$ & $\begin{array}{l}\lambda_{\mathrm{em}} \\
(\mathrm{nm})\end{array}$ & $K_{11}\left(\mathrm{M}^{-1}\right)$ & $K_{12}\left(\mathrm{M}^{-1}\right)$ & $\tau(\mathrm{ns})$ & $\Phi_{\mathrm{F}}$ & $\begin{array}{l}\varepsilon_{\max } \times \Phi_{\mathrm{F}} \\
\left(10^{3} \mathrm{M}^{-1} \mathrm{~cm}^{-1}\right)\end{array}$ \\
\hline sG4 & 699 & 114 & 722 & $8.1 \times 10^{6}$ & - & 5.8 & $0.74 \pm 0.07$ & 84 \\
\hline VAV-1 & 699 & 118 & 722 & $1.2 \times 10^{7}$ & - & 5.6 & $0.67 \pm 0.02$ & 79 \\
\hline c-myc & 699 & 108 & 721 & $1.0 \times 10^{7}$ & - & 5.5 & $0.52 \pm 0.03$ & 56 \\
\hline $25 \mathrm{Ceb}$ & 698 & 108 & 719 & $9.0 \times 10^{5}$ & - & 6.0 & $0.66 \pm 0.01$ & 71 \\
\hline VEGF & 699 & 113 & 721 & $1.6 \times 10^{2}$ & $3.7 \times 10^{9}$ & 5.7 & $0.74 \pm 0.08$ & 84 \\
\hline Kras 22 & 699 & 116 & 721 & $1.5 \times 10^{7}$ & $1.7 \times 10^{6}$ & 5.4 & - & - \\
\hline Kras 32 & 699 & 103 & 721 & $5.9 \times 10^{6}$ & $4.1 \times 10^{5}$ & 5.6 & - & - \\
\hline bcl-2 & 699 & 113 & 721 & 36 & $6.0 \times 10^{8}$ & - & - & - \\
\hline ckit-87up & 699 & 103 & 721 & $3.1 \times 10^{2}$ & $4.3 \times 10^{8}$ & 5.5 & - & - \\
\hline ckit-2 & 699 & 92 & 720 & $5.0 \times 10^{2}$ & $4.0 \times 10^{8}$ & - & - & - \\
\hline
\end{tabular}

${ }^{a}$ Data fitting with $1: 1$ and 1:2 models was performed according to ref. 28 and the software provided at the website http://supramolecular.org. We like to note that the credibility of the derived values for the $1: 2$ case is lower than for the $1: 1$ case. This is due to the presence of three species and unknown fluorescence quantum yields of the $1: 1$ and the $1: 2$ complexes, and changing amounts of these complexes during the titration experiment. To give the same weight to UV/Vis and fluorescence experiments we evaluated both titrations independently and took the average values. 
thus in a more efficient rigidification as desired for a fluorescence increase. On the other hand, the negative effect of the external quartets surrounded by loops in antiparallel G4s such as 22AG and c-kit hampers to some extent the end-stacking ability of CAS-C1 inducing a lower overall optical response. The aforementioned rationale is confirmed by using the closely related c-myc G4 analogue sG4, featuring no flanking overhang residues at both $5^{\prime}$ and $3^{\prime}$ ends that limit the steric hindrance, to enhance the light-up ability of CAS-C1. Indeed, complexation of the squaraine with SG4 provides a $\Phi_{\mathrm{F}}$ of 0.74 which is among the highest values reported for nucleic acid complexes within comparable spectral range (Table 1$).^{30,31}$ Structural insights into the binding mode of CAS-C1 with G4s were provided by docking binding analysis on the G4s whose structures in solution had been derived by NMR studies under similar experimental conditions. The elucidated structures of G4s are referenced in Table $\mathrm{S} 1 \dagger$. In the case of c-myc, the squaraine chromophore is the recognition unit leading to a perfect complementary match with the $3^{\prime}$-quartet (Fig. 3c). However, the substitution of side groups at the squaraine chromophore has surely an impact in the binding. On one side, the massive number of glycol chains are responsible for the enhanced interaction compared to SQgl by establishing strong electrostatic interactions with the polar backbone of the G4. Presumably, the glycol chains displace water molecules from the grooves and thereby provide an entropic contribution to the stability of the G4-CAS-C1 complex, especially for the parallel ones, ${ }^{32}$ which explains the strengthening of the binding affinity compared with SQgl. On the other hand, it is reasonable to assume that the steric hindrance of the benzyloxy unit, which is accommodated in the hydrophobic pockets in the side of the quartet (Fig. 3c and d), largely contribute to the selectivity of the interaction.

\section{Two-photon absorption}

A fundamental optical limitation is encountered at the nanoscale level owing to Abbe's law of diffraction, which limits the maximum spatial resolution to approximately half the wavelength of light used to image the specimen. ${ }^{33}$ One way to overcome this limitation involves the use of nonlinear optical procedures based on multi-photon absorption processes. In this approach, the excitation of a chromophore is performed by the simultaneous absorption of two-photons with half the energy required for the corresponding one-photon absorption process. As a result, the excitation wavelength is confined in the first and second NIR biological transparency windows where the diffusion and absorption of light by inherently biological matrices is minimized, and thus allowing deep tissue penetration. Moreover, the low-energy excitation prevents photobleaching and cellular damages in biological samples. Importantly, the TPA is limited to a small femtoliter volume and occurs only at the focal point of a focused laser beam resulting in 3D image resolution. Despite the lower energy of the IR light compared to visible and UV light, this excitation mode has been proven to induce damage to the samples when using classical fluorophore that possess low TPA cross-section $\left(\sigma_{2}<50 \mathrm{GM}\right) \cdot{ }^{34} \mathrm{It}$ turns out that, besides having significant photoluminescence properties in the NIR, a chromophore should also possess high $\sigma_{2}$ values to be considered as a suitable probe for two-photon microscopy procedures in NIR-to-NIR configurations. The TPA spectra of CAS-C1 complexed with parallel G4s including VAV1, VEGF and c-myc were measured by two-photon excited fluorescence (TPEF) method in the range 1000-1550 $\mathrm{nm}$ and are reported in Fig. 4 as $\sigma_{2} \times \Phi_{\mathrm{F}}$.

In general, all the complexes show similar TPA spectra composed of an intense high energy TPA band blue shifted compared to the one-photon transition energy and a weak low energy TPA band that matches well the wavelength-doubled 1PA spectra. These spectral features are usually associated with centrosymmetric quadrupolar molecules, in which selection rules for $1 \mathrm{PA}$ and 2PA electronic transitions are mutually exclusive. CAS-C1 presents a non-centrosymmetric character with a quasi- $C_{2 \mathrm{v}}$ symmetry and can be treated as bent quadrupolar chromophore, of which the quadrupolar conformation gives rise to an intense hypsochromically shifted TPA while the residual dipolar character results in a weak TPA transition overlapped with the 1PA maximum. Similar conclusions were recently reported for extended Michler's ketone, heptamethine, diarylboryl and anthracenyl derivatives. ${ }^{35-38}$ The TPA crosssection $\left(\sigma_{2}\right)$ values for the CAS-C1:G4 systems follow the order: CAS-C1 : VAV1 $\sigma_{2}=312$ GM, CAS-C1 : VEGF $\sigma_{2}=278 \mathrm{GM}$ and CAS-C1 : c-myc $\sigma_{2}=273 \mathrm{GM}$ at $1275 \mathrm{~nm}$ (Fig. S25 $\dagger$ ). Interestingly, the CAS-C1 aggregate also shows TPA absorption close to half energy of the corresponding OPA, in agreement with a centrosymmetric aggregate (Fig. S26†).

The high values of $\sigma_{2}$ combined with the associated 2PA maximum are an optimal combination for NIR-to-NIR configurations since excitation of CAS-C1 : G4 systems can be performed within the NIR-II window by TPA $\left(\lambda_{\operatorname{ex}(2 \mathrm{PA})}=1275 \mathrm{~nm}\right)$

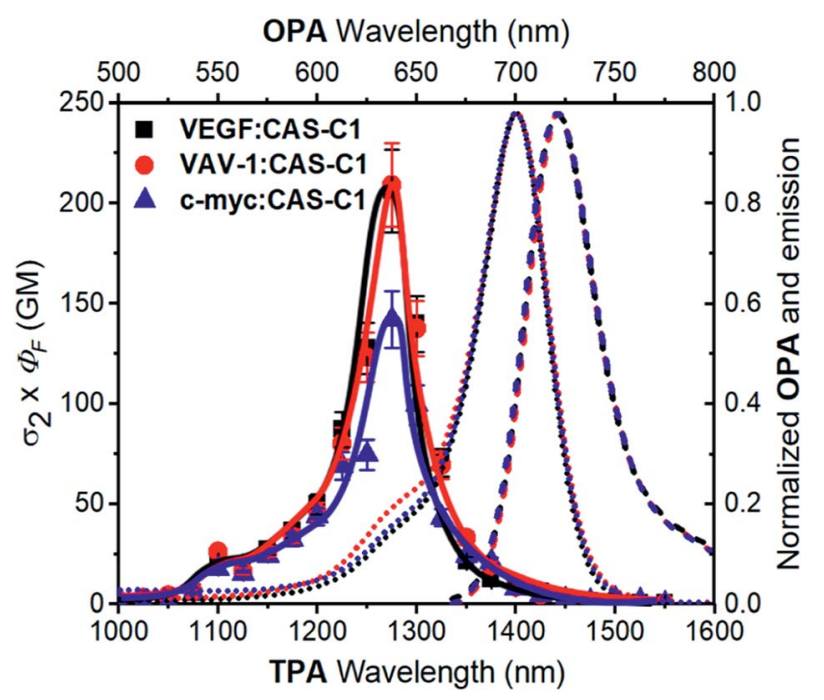

Fig. 4 Two-photon molecular brightness $\left(\sigma_{2} \times \Phi_{\mathrm{F}}\right)$ plot of CAS-C1 in the presence of VEGF, VAV-1 and c-myc $\left(10 \mathrm{mM}\right.$ Tris, $100 \mathrm{mM} \mathrm{K}^{+}, \mathrm{pH}$ 7.2) at molar ratio $(r)=7$. The values of the $\Phi_{\mathrm{F}}$ used are tabulated in Table 1. As a comparison, the OPA corresponding to the double of the energy of the TPA is shown (top and right axis): the absorption (dotted lines) and emission (dashed line) spectra corresponding to the CAS-C1 complexes with the G4s are shown. 
with emissions centered within the NIR-I spectral region $\left(\lambda_{\mathrm{em}} \approx 720 \mathrm{~nm}\right)$. In line with the trend depicted for $\sigma_{2}$ also the two-photon molecular brightness $\left(\sigma_{2} \times \Phi_{\mathrm{F}}\right)$, as a result of the strong fluorescence enhancement of CAS-C1 bound to parallel G4s, follow the same order (i.e. CAS-C1 : VAV1 $\sigma_{2} \times \Phi_{\mathrm{F}}=209$ GM, CAS-C1 : VEGF $\sigma_{2} \times \Phi_{\mathrm{F}}=206 \mathrm{GM}$ and CAS-C1 : c-myc $\sigma_{2} \times \Phi_{\mathrm{F}}=142 \mathrm{GM}$ at $1275 \mathrm{~nm}$ ) indicating the suitability of CAS-C1 to be used as a nonlinear biomarker for parallel G4 detection by using ultrasensitive fluorescence microscopy techniques. These values are nevertheless lower than those observed for monomeric CAS-C1 in organic solvent $\left(\sigma_{2} \times \Phi_{\mathrm{F}}=386 \mathrm{GM}\right.$ in isopropanol, Fig. S24†). This result is in agreement with recent results from Goodson and co-workers ${ }^{39}$ and some of $\mathrm{us}^{\mathbf{4 0 , 4 1}}$ that relate the changes in the TPA crosssection to the different DNA binding modes. In particular, groove binders exhibit an enhanced TPA cross-section due to the DNA electric field induced enhancement of the transition dipole moment, while intercalators or end-stackers show a decrease in the TPA cross-section when complexed with DNA. Accordingly, this finding also supports our proposal that CASC1 binds to parallel G4s mainly via end-stacking mode in agreement with the docking results. The end-stacking ability of CAS-C1 was further confirmed through electronic circular dichroism (ECD) measurements (Fig S27 $\dagger$ ). ${ }^{42}$ Indeed, the native conformation of the parallel G4s was almost not affected by the presence of the squaraine dye indicating no or very weak perturbation on the quadruplex morphologies. By analogy with previously reported data for a G4 intercalator, ${ }^{\mathbf{4 1}}$ the absence of conformational changes observed for CAS-C1-parallel G4 systems constitutes a clear evidence of the ability of CAS-C1 to coordinate the external G-tetrads, ruling out the possible intercalative binding behavior of our probe known to give rise to pronounced structural changes associated with a partially disruption of the stacking interactions that govern G4 formation.

\section{Conclusions}

In conclusion, the herein reported results on a new squaraine dye CAS-C1 with outstanding binding strength and selectivity towards parallel G4 motif open up a new research line for this fascinating class of NIR fluorophores and functional dyes ${ }^{\mathbf{1 7 , 4 3}}$ beyond previous applications in organic electronics, ${ }^{44}$ photovoltaics $^{45}$ and exciton transport. ${ }^{46}$ The CAS-C1 probe once complexed with parallel G4s exhibits a NIR light-up fluorescence response with quantum yields that go beyond 0.7. As a result, close-to-optimal one-photon molecular brightness in the far red-NIR region can be obtained. The parallel G4 preference of CAS-C1 is accompanied by binding constants that exceed by two-orders of magnitude those calculated for the previously reported squaraine SQgI. The selectivity of the probe is tested toward a large panel of anti-parallel G4s and singleand double-stranded morphologies of any size and composition making CAS-C1 a highly discriminatory parallel G4-binder. The docking results showed that the squaraine chromophore bestows the necessary selectivity for the interactions with the G4s. The molecular design of CAS-C1 allowed us, on the one hand, to increase the selectivity of the interaction by exploiting the more easily accessible G-quartet surface of parallel G4s and, on the other hand, the massive presence of glycol chains in the hydrophobic pocket of the G4 imparted strong affinity. The complexes of CAS-C1 with parallel G4s also possess strong twophoton absorption with maxima centered at $1275 \mathrm{~nm}$, which make them ideally suited for NIR-to-NIR imaging procedures. Ultimately, the enhanced fluorescence quantum yields and the two-photon absorption cross-section enable one to obtain exceptionally high two-photon molecular brightness values within the NIR-II window that are desired for virtually all ultrasensitive fluorescence spectroscopy and microscopy techniques. These overall findings award CAS-C1 as the best twophoton parallel G4-specific probe currently available.

\section{Conflicts of interest}

There are no conflicts to declare.

\section{Acknowledgements}

We thank Prof. Pall Thordarson (Sydney, Australia) for his helpful advice for the analysis of the titration experiments. The research at the University of Würzburg was financially supported by the State of Bavaria with the establishment of the KeyLab for Supramolecular Polymers of the Bavarian Polymer Institute (BPI). At the Wroclaw University of Science and Technology, financial support from a statutory activity subsidy from the Polish Ministry of Science and Higher Education for the Faculty of Chemistry of WUST, KNOW - center of excellence in biotechnology project, and Harmonia grant DEC-2016/22/M/ ST4/00275 are highly acknowledged.

\section{Notes and references}

1 P. Murat and S. Balasubramanian, Curr. Opin. Genet. Dev., 2014, 25, 22-29.

2 R. Hänsel-Hertsch, M. Di Antonio and S. Balasubramanian, Nat. Rev. Mol. Cell Biol., 2017, 18, 279-284.

3 A. M. Fleming, J. Zhu, Y. Ding, J. A. Visser, J. Zhu and C. J. Burrows, Biochemistry, 2018, 57, 991-1002.

4 A. Bedrat, L. Lacroix and J.-L. Mergny, Nucleic Acids Res., 2016, 44, 1746-1759.

5 P. Williams, L. Li, X. Dong and Y. Wang, J. Am. Chem. Soc., 2017, 139, 12426-12429.

6 S. Zhang, Y. Wu and W. Zhang, ChemMedChem, 2014, 9, 899911.

7 T. M. Ou, Y. J. Lu, J. H. Tan, Z. S. Huang, K. Y. Wong and L. Q. Gu, ChemMedChem, 2008, 3, 690-713.

8 S. Balasubramanian, L. H. Hurley and S. Neidle, Nat. Rev. Drug Discovery, 2011, 10, 261-275.

9 Q. Cao, Y. Li, E. Freisinger, P. Z. Qin, R. K. O. Sigel and Z.-W. Mao, Inorg. Chem. Front., 2017, 4, 10-32.

10 L. Tuong Vy Thi, H. Sujin, C. Junghyun and P. Hyun-Ju, Curr. Pharm. Des., 2012, 18, 1948-1972. 
11 A. Garci, K. J. Castor, J. Fakhoury, J.-L. Do, J. Di Trani, P. Chidchob, R. S. Stein, A. K. Mittermaier, T. Friščić and H. Sleiman, J. Am. Chem. Soc., 2017, 139, 16913-16922.

12 M. Wang, Z. Mao, T.-S. Kang, C.-Y. Wong, J.-L. Mergny, C.-H. Leung and D.-L. Ma, Chem. Sci., 2016, 7, 2516-2523.

13 K.-H. Leung, H.-Z. He, B. He, H.-J. Zhong, S. Lin, Y.-T. Wang, D.-L. Ma and C.-H. Leung, Chem. Sci., 2015, 6, 2166-2171.

14 A. C. Bhasikuttan and J. Mohanty, Chem. Commun., 2015, 51, 7581-7597.

15 B. R. Vummidi, J. Alzeer and N. W. Luedtke, ChemBioChem, 2013, 14, 540-558.

16 Z. Li and K. B. Grant, RSC Adv., 2016, 6, 24617-24634.

17 J. Zhao, D. Zhong and S. Zhou, J. Mater. Chem. B, 2018, 6, 349-365.

18 V. Grande, F. Doria, M. Freccero and F. Würthner, Angew. Chem., Int. Ed., 2017, 56, 7520-7524.

19 B. Jin, X. Zhang, W. Zheng, X. J. Liu, J. Zhou, N. Zhang, F. Y. Wang and D. H. Shangguan, Anal. Chem., 2014, 86, 7063-7070.

20 X. Zhang, Y. Wei, T. Bing, X. Liu, N. Zhang, J. Wang, J. He, B. Jin and D. Shangguan, Sci. Rep., 2017, 7, 4766.

21 M. Zuffo, A. Guédin, E.-D. Leriche, F. Doria, V. Pirota, V. Gabelica, J.-L. Mergny and M. Freccero, Nucleic Acids Res., 2018, DOI: 10.1093/nar/gky607.

22 A. Laguerre, Y. Chang, M. Pirrotta, N. Desbois, C. P. Gros, E. Lesniewska and D. Monchaud, Org. Biomol. Chem., 2015, 13, 7034-7039.

23 F. Cuenca, O. Greciano, M. Gunaratnam, S. Haider, D. Munnur, R. Nanjunda, W. D. Wilson and S. Neidle, Bioorg. Med. Chem. Lett., 2008, 18, 1668-1673.

24 U. Mayerhöffer, M. Gsänger, M. Stolte, B. Fimmel and F. Würthner, Chem.-Eur. J., 2013, 19, 218-232.

25 T. Brixner, R. Hildner, J. Köhler, C. Lambert and F. Würthner, Adv. Energy Mater., 2017, 7, 1700236.

26 F. Würthner, Acc. Chem. Res., 2016, 49, 868-876.

27 L. K. S. von Krbek, C. A. Schalley and P. Thordarson, Chem. Soc. Rev., 2017, 46, 2622-2637.

28 D. B. Hibbert and P. Thordarson, Chem. Commun., 2016, 52, 12792-12805.

29 P. Thordarson, Chem. Soc. Rev., 2011, 40, 1305-1323.

30 W. Xu, K. M. Chan and E. T. Kool, Nat. Chem., 2017, 9, 10431055.

31 Y. V. Suseela, N. Narayanaswamy, S. Pratihar and T. Govindaraju, Chem. Soc. Rev., 2018, 47, 1098-1131.
32 D. Miyoshi, H. Karimata and N. Sugimoto, J. Am. Chem. Soc., 2006, 128, 7957-7963.

33 J. Moreno, M. Gerecke, L. Grubert, S. A. Kovalenko and S. Hecht, Angew. Chem., Int. Ed., 2016, 55, 1544-1547.

34 B. Dumat, G. Bordeau, E. Faurel-Paul, F. Mahuteau-Betzer, N. Saettel, G. Metge, C. Fiorini-Debuisschert, F. Charra and M.-P. Teulade-Fichou, J. Am. Chem. Soc., 2013, 135, 1269712706.

35 N. S. Makarov, S. Mukhopadhyay, K. Yesudas, J. L. Brédas, J. W. Perry, A. Pron, M. Kivala and K. Müllen, J. Phys. Chem. A, 2012, 116, 3781-3793.

36 Q. Zou, Y. Zhao, N. S. Makarov, J. Campo, H. Yuan, D. C. Fang, J. W. Perry and F. Wu, Phys. Chem. Chem. Phys., 2012, 14, 11743-11752.

37 S. Pascal, S. Denis-Quanquin, F. Appaix, A. Duperray, A. Grichine, B. L. Guennic, D. Jacquemin, J. Cuny, S. H. Chi, J. W. Perry, B. V. D. Sanden, C. Monnereau, C. Andraud and O. Maury, Chem. Sci., 2017, 8, 381394.

38 B. Mettra, F. Appaix, J. Olesiak-Banska, T. Le Bahers, A. Leung, K. Matczyszyn, M. Samoc, B. van der Sanden, C. Monnereau and C. Andraud, ACS Appl. Mater. Interfaces, 2016, 8, 17047-17059.

39 P. H. Doan, D. R. G. Pitter, A. Kocher, J. N. Wilson and T. Goodson, J. Am. Chem. Soc., 2015, 137, 9198-9201.

40 M. Deiana, B. Mettra, K. Matczyszyn, D. Pitrat, J. OlesiakBanska, C. Monnereau, C. Andraud and M. Samoc, Biomacromolecules, 2016, 17, 3609-3618.

41 M. Deiana, B. Mettra, L. Martinez-Fernandez, L. M. Mazur, K. Pawlik, C. Andraud, M. Samoc, R. Improta, C. Monnereau and K. Matczyszyn, J. Phys. Chem. Lett., 2017, 8, 5915-5920.

42 R. del Villar-Guerra, J. O. Trent and J. Chaires, Angew. Chem., Int. Ed., 2018, 57, 7171-7175.

43 A. Ajayaghosh, Acc. Chem. Res., 2005, 38, 449-459.

44 M. Gsänger, E. Kirchner, M. Stolte, C. Burschka, V. Stepanenko, J. Pflaum and F. Würthner, J. Am. Chem. Soc., 2014, 136, 2351-2362.

45 U. Mayerhöffer, K. Deing, K. Gruß, H. Braunschweig, K. Meerholz and F. Würthner, Angew. Chem., Int. Ed., 2009, 48, 8776-8779.

46 C. Lambert, F. Koch, S. F. Völker, A. Schmiedel, M. Holzapfel, A. Humeniuk, M. I. S. Röhr, R. Mitric and T. Brixner, J. Am. Chem. Soc., 2015, 137, 7851-7861. 